University of Massachusetts Amherst

ScholarWorks@UMass Amherst

Publications

MURI on Photomechanical Materials

2021

\title{
Light-induced stress and work in photomechanical materials
}

\author{
Anastasiia Svanidze \\ Kent State University \\ Tianyi Guo \\ Kent State University \\ Xiaoyu Zheng \\ Kent State University \\ Peter Palffy-Muhoray \\ Kent State University
}

Follow this and additional works at: https://scholarworks.umass.edu/muri_pubs

Svanidze, Anastasiia; Guo, Tianyi; Zheng, Xiaoyu; and Palffy-Muhoray, Peter, "Light-induced stress and work in photomechanical materials" (2021). Physical Review E. 36.

https://doi.org/10.1103/PhysRevE.103.L051002

This Article is brought to you for free and open access by the MURI on Photomechanical Materials at ScholarWorks@UMass Amherst. It has been accepted for inclusion in Publications by an authorized administrator of ScholarWorks@UMass Amherst. For more information, please contact scholarworks@library.umass.edu. 


\title{
Light-induced stress and work in photomechanical materials
}

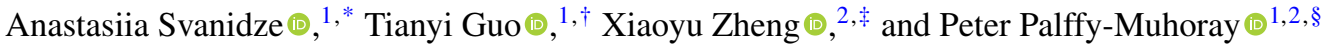 \\ ${ }^{1}$ Advanced Materials and Liquid Crystal Institute, Kent State University, Ohio 44242, USA \\ ${ }^{2}$ Department of Mathematical Sciences, Kent State University, Ohio 44242, USA
}

(Received 15 February 2021; accepted 5 May 2021; published 24 May 2021)

\begin{abstract}
Increasingly important photomechanical materials produce stress and mechanical work when illuminated. We propose experimentally accessible performance metrics for photostress and photowork, enabling comparison of materials performance. We relate these metrics to material properties, providing a framework for the design and optimization of photomechanical materials.
\end{abstract}

DOI: 10.1103/PhysRevE.103.L051002

\section{INTRODUCTION}

The availability of high-intensity light sources and the ease of optical power transmission have generated considerable interest in materials capable of directly exerting forces and performing mechanical work under the influence of light. Such photomechanical (PM) materials exhibit stresses on illumination, can undergo shape changes, and can perform mechanical work. Despite this interest, there are no standard methods and criteria in use today for assessing materials performance in producing photostress or performing photowork. Materials of particular interest are liquid crystal elastomers $[1,2]$ and networks $[3,4]$, semicrystalline polymers $[5,6]$, and organic crystals [7-9].

We propose here performance criteria that can be evaluated using results from experiments and which enable comparison of materials performance. We further establish the dependence of these criteria on material parameters, opening the door toward systematic optimization of PM materials.

\section{PHOTOSTRESS}

The first challenge in establishing the relation between the light-induced photostress $\sigma^{\mathrm{ph}}$ and the incident light is the identification of an appropriate descriptor of the light. PM materials are typically anisotropic, with a polarization, intensity, and wavelength-dependent photoresponse. In addition to intensity, the descriptor thus needs to carry information about polarization and frequency; it also needs to be a tensor.

Although interestingly the Minkowski stress does not carry polarization information, its first term, the outer product of the electric field and the electric displacement of light, does, and hence can serve in this role. We denote it as $\boldsymbol{\sigma}^{l}=\mathbf{E D}$, the light stress. This is simply related to the light intensity $I=\frac{1}{2} c \operatorname{Tr}\left(\sigma^{l}\right)$, where $c$ is the speed of light, and $\operatorname{Tr}$ is the trace.

\footnotetext{
*asvanidz@kent.edu

†tguo2@kent.edu

${ }^{\ddagger}$ xzheng3@kent.edu

${ }^{\S}$ Corresponding author: mpalffy@kent.edu
}

In the linear regime, one expects the constitutive relation

$$
\boldsymbol{\sigma}^{\mathrm{ph}}=\mathbf{R}: \boldsymbol{\sigma}^{l},
$$

where $\mathbf{R}$ is a fourth-rank tensor representing response, the stress (or momentum current density) gain. The operator : sums over two repeated indices, that is $\mathbf{A}: \mathbf{B}=A_{i j k l} B_{k l}$. The dimensionless elements of $\mathbf{R}$ quantify the efficiency of PM materials in directly producing stress from light and enable comparison of materials performance. For materials with high symmetry, such as transversely isotropic materials, $\mathbf{R}$ contains relatively few independent elements whose experimental determination is feasible.

If the stresses are symmetric and the sample is uniaxial, one can write

$$
\left[\begin{array}{c}
\sigma_{11}^{\mathrm{ph}} \\
\sigma_{22}^{\mathrm{ph}} \\
\sigma_{33}^{\mathrm{ph}} \\
\sigma_{23}^{\mathrm{ph}} \\
\sigma_{13}^{\mathrm{ph}} \\
\sigma_{12}^{\mathrm{ph}}
\end{array}\right]=\left[\begin{array}{cccccc}
R_{11} & R_{12} & R_{13} & 0 & 0 & 0 \\
R_{21} & R_{22} & R_{23} & 0 & 0 & 0 \\
R_{31} & R_{32} & R_{33} & 0 & 0 & 0 \\
0 & 0 & 0 & R_{44} & 0 & 0 \\
0 & 0 & 0 & 0 & R_{55} & 0 \\
0 & 0 & 0 & 0 & 0 & R_{66}
\end{array}\right]\left[\begin{array}{c}
\sigma_{11}^{l} \\
\sigma_{22}^{l} \\
\sigma_{33}^{l} \\
\sigma_{23}^{l} \\
\sigma_{13}^{l} \\
\sigma_{12}^{l}
\end{array}\right],
$$

where in general not all of the matrix elements are independent. By using light linearly polarized along a principal axis, the diagonal elements of $\mathbf{R}$ can be determined.

We next inquire about the connection between the elements of $\mathbf{R}$ and material parameters of PM samples.

To gain some insight, we make use of the following elementary model. We assume that PM bulk materials consist of a solid elastic host, in which discrete photoisomerizable "dye" molecules with volume $v_{d}$ are embedded randomly in position with uniform average density. When illuminated, a dye molecule in the low-energy isomer conformation absorbs a single photon with energy $h v_{d}$, and undergoes a prescribed volume-conserving shape-changing transformation, such as from a low-energy prolate ellipsoid to a sphere, the highenergy isomer conformation. The dye shape change can be described by a bare dye strain $\varepsilon_{0}^{d}$; see Fig. 1 . In the absence 
low-energy isomer

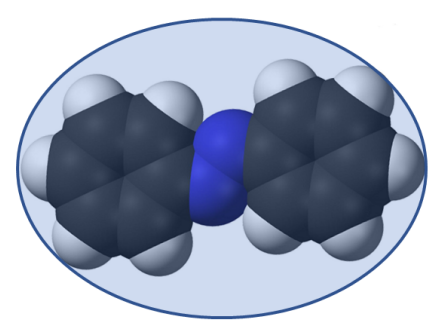

trans- high-energy isomer

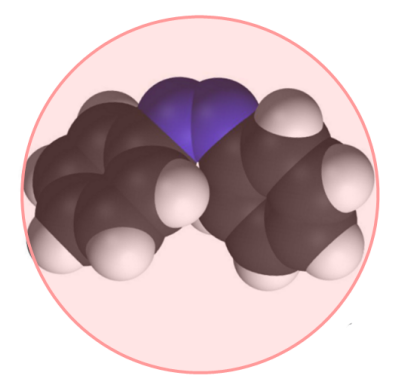

cis-
FIG. 1. A schematic shows the shape change of a dye isomer under illumination which is excited from the low energy "trans" (left) to the high energy "cis" (right) conformation. The bare strain tensor can be calculated based on the change of semiaxes of the ellipsoid.

of an externally applied stress, in an ideal continuum model where identical solid inclusions undergo a volume-conserving strain $\varepsilon_{0}^{d}$ in an elastic solid host, the resulting bulk photo strain may be assumed to be $\varepsilon^{\mathrm{ph}}=\phi_{h d} \varepsilon_{0}^{d}$, where $\phi_{h d}$ is the volume fraction of the high-energy conformers. However, the orientations of the dye molecules are not identical; the relevant quantity is their orientational average $\left\langle\varepsilon_{0}^{d}\right\rangle=S_{d} \varepsilon_{0}^{d}$ where $-\frac{1}{2} \leqslant S_{d} \leqslant 1$ is the orientational order parameter. In addition, interactions with the host can further modify this, ${ }^{1}$ and we write instead $\boldsymbol{\varepsilon}^{\mathrm{ph}}=\phi_{h d} \varepsilon^{d}$, where $\boldsymbol{\varepsilon}^{d}=\beta S_{d} \varepsilon_{0}^{d}$ is the effective dye strain, and $\beta$ is a constant. The corresponding photostress $\sigma^{\mathrm{ph}}$, measured at zero bulk strain, is assumed to be

$$
\boldsymbol{\sigma}^{\mathrm{ph}}=\phi_{h d} \mathbf{C}: \boldsymbol{\varepsilon}^{d},
$$

where $\mathbf{C}$ is the stiffness tensor of the host.

The photoinduced high-energy conformers relax back into the low-energy conformation after a lifetime $\tau$. For simplicity, we assume that the high-energy conformer always has enough energy to guarantee the strain $\boldsymbol{\varepsilon}^{d}$, and we assume that $\tau$ is a constant regardless of the environment. In the photostationary state, the decay rate of the high energy isomer population must equal the creation rate from the low energy isomer population with volume fraction $\phi_{l d}$, and

$$
\frac{\phi_{h d}}{\phi_{l d}}=\left(\sigma^{d}\right)^{-1}: \sigma^{l},
$$

where

$$
\left(\boldsymbol{\sigma}^{d}\right)^{-1}=\frac{c \tau}{h v_{d}} \mathbf{a}^{l d}
$$

\footnotetext{
${ }^{1}$ In the simplest case, $\beta=1$, but in general, it can be reduced or increased due to the interaction of the dye and the host. Examples would be free volume effects in the host reducing the effective bare dye strain, or order parameter change of a liquid crystal host, reducing anisotropic dispersion interaction due to photoisomerization of the dye; coupling of the order parameter to the polymer network results in bulk strain. Reference [10] reports values of $\varepsilon^{d}>1$.
}

and $\mathbf{a}^{l d}$ is the absorption cross-section tensor of the lowenergy conformer. ${ }^{2}$ We note that dye stress $\sigma^{d}$, with the units of energy current density, is a material property. Since the total dye volume fraction $\phi_{d}=\phi_{h d}+\phi_{l d}$ is a constant,

$$
\phi_{h d}=\phi_{d} \frac{\left(\sigma^{d}\right)^{-1}: \sigma^{l}}{1+\left(\sigma^{d}\right)^{-1}: \sigma^{l}},
$$

indicating nonlinearity and saturation when $\left(\sigma^{d}\right)^{-1}: \sigma^{l} \gtrsim 1$.

The photostress, in the linear regime, from Eqs. (3) and (4), is

$$
\boldsymbol{\sigma}^{\mathrm{ph}}=\phi_{d}\left(\mathbf{C}: \boldsymbol{\varepsilon}^{d}\right)\left[\left(\boldsymbol{\sigma}^{d}\right)^{-1}: \boldsymbol{\sigma}^{l}\right],
$$

and the stress gain function $\mathbf{R}$, from Eqs. (1) and (8), is

$$
\mathbf{R}=\phi_{d}\left(\mathbf{C}: \boldsymbol{\varepsilon}^{d}\right)\left(\boldsymbol{\sigma}^{d}\right)^{-1} .
$$

The dependence of the stress gain $\mathbf{R}$ on material parameters is a key result. Its constituents are more readily seen in an isotropic system; there Eq. (9) can be written as $R=\phi_{d} \varepsilon^{d} Y c / I_{0}$, where $Y$ is Young's modulus, and $I_{0}=$ $h v_{d} /\left(a^{l d} \tau\right)$. According to Eq. (9), our model indicates that, for a given light intensity, photostress is proportional to the corresponding elastic modulus [11] of PM materials, in agreement with the experimental observations in Fig. 2.

The above result can be compared with experiment. By illuminating samples with light polarized along the symmetry axis $\hat{\mathbf{z}}$ of uniaxial liquid crystal elastomers and other transversely isotropic samples, the element $R_{z z}=\sigma_{z z}^{\mathrm{ph}} c / I$ can be determined. Measuring stress gain in bels, the quantity $R_{z z}^{\prime}=\log _{10} R_{z z}$, as a function of Young's modulus, is shown in Fig. 2, demonstrating the experimentally observed stiffness dependence of the response.

\section{PHOTOWORK}

We next consider the efficiency of PM materials in converting light energy to mechanical work. We define the efficiency $\Gamma$ as the ratio of mechanical work done on a load to the energy absorbed from light. Experimental determination of $\Gamma$ is usually straightforward. To relate the efficiency to material parameters, we make use of the relation between photostress and light in Eqs. (1) and (9).

When a PM material is illuminated, photoisomerizable dye molecules photoisomerized into the high-energy conformation exhibit a prescribed strain $\boldsymbol{\varepsilon}^{d}$, leading to bulk photostress and photostrain. If there are no externally applied stresses, the sample deforms, and mechanical work produced by light is stored as elastic energy. If an external load is present providing an external stress, then the mechanical work enabled by light is partitioned between work on the load and elastic energy stored in the PM host. To simply illustrate this, we consider an illuminated isotropic elastomer host without any external

\footnotetext{
${ }^{2}$ We take the this to be

$$
\mathbf{a}^{l d}=\frac{2 \pi}{\varepsilon_{0} \lambda} \operatorname{Im} \alpha^{l d},
$$

where $\operatorname{Im} \boldsymbol{\alpha}^{l d}$ is the imaginary part of the polarizability tensor, $\varepsilon_{0}$ is the permittivity of free space, and $\lambda$ is the wavelength.
} 


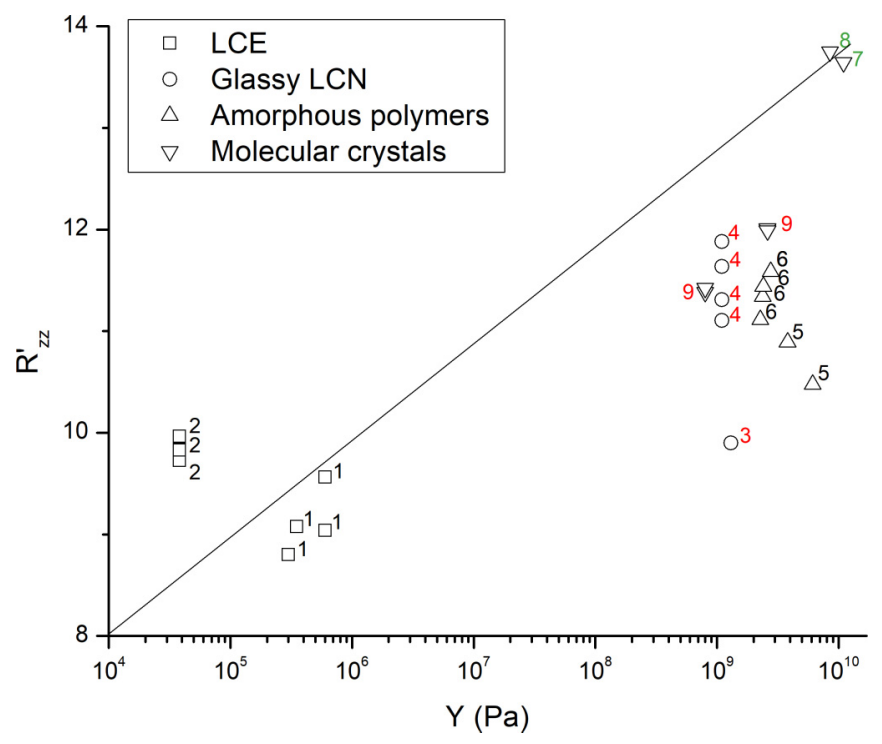

FIG. 2. Graph of $R_{z z}^{\prime}$ vs. Young's modulus $Y$. The numbers on the graph indicate that $R_{z z}^{\prime}$ is calculated from data published in papers corresponding to the reference number next to each point in the graph. The line indicates proportionality; deviations are ascribed to differences in values of $\phi_{l d}, \varepsilon^{d}$, and $I_{0}$.

stress, but with a volume conserving uniaxial photostrain and corresponding uniaxial photostress. The stored elastic energy density in a volume conserving material is then, with $\varepsilon^{\mathrm{ph}}=$ $\varepsilon_{z z}^{\mathrm{ph}} \operatorname{diag}(-1 / 2,-1 / 2,1)$

$$
\mathcal{E}_{s t}=G \boldsymbol{\varepsilon}^{\mathrm{ph}}: \boldsymbol{\varepsilon}^{\mathrm{ph}}=\frac{3}{2} G\left(\varepsilon_{z z}^{\mathrm{ph}}\right)^{2}=\frac{3}{8} \frac{1}{G}\left(\sigma_{z z}^{\mathrm{ph}}\right)^{2},
$$

where $G$ is the shear modulus and $\varepsilon_{z z}$ is element of the bulk strain. If a load is present, with a single stress component $\sigma_{z z}^{\text {load }}$, the stored energy becomes

$$
\mathcal{E}_{s t}=\frac{3}{8} \frac{1}{G}\left(\sigma_{z z}^{\mathrm{ph}}-\frac{2}{3} \sigma_{z z}^{\text {load }}\right)^{2} .
$$

If the load stress is written in terms of the photostress as $\sigma_{z z}^{\text {load }}=\gamma \sigma_{z z}^{\mathrm{ph}}$, where $\gamma<1$, then the stored elastic energy density becomes

$$
\mathcal{E}_{s t}=\frac{1}{2} Y\left(\varepsilon_{z z}^{\mathrm{ph}}\right)^{2}\left(1-\frac{2}{3} \gamma\right)^{2},
$$

where $Y=3 G$ is Young's modulus, while the work density $\mathcal{W}$ done on the load is

$$
\mathcal{W}=\sigma_{z z}^{\text {load }} \varepsilon_{z z}=\frac{1}{2} Y\left(\varepsilon_{z z}^{\mathrm{ph}}\right)^{2} \frac{4}{3} \gamma\left(1-\frac{2}{3} \gamma\right) .
$$

The photowork done on the external load thus depends on the ratio $\gamma$ of the load stress to the photostress; it is maximized here when an impedance match function, here $f(\gamma)=\frac{4}{3} \gamma\left(1-\frac{2}{3} \gamma\right)$, is a maximum. When $\gamma=3 / 4, \sigma_{z z}^{\text {load }}=$ $3 / 4 \sigma_{z z}^{\mathrm{ph}}=1 / 2 Y \varepsilon_{z z}^{\mathrm{ph}}$.

In general, the mechanical work density done by the light on the sample is

$$
\mathcal{W}=\frac{1}{2} \phi_{h d}^{2} \varepsilon^{d}: \mathbf{C}: \boldsymbol{\varepsilon}^{d} f(\gamma)
$$

where $f(\gamma)$ may differ from the expression above, but we expect its argument $\gamma$ to remain the same (the ratio of the load stress to the photostress), and its maximum to occur when $\gamma$ is of order unity.

The system is assumed to be overdamped; the time for work is taken to be the characteristic response time $t_{r}$ of the system. ${ }^{3}$ The energy input per volume from the absorbed light in time $t_{r}$ is

$$
\mathcal{E}_{\text {in }}=\frac{\phi_{l d}}{v_{d}} c \mathbf{a}^{l d}: \boldsymbol{\sigma}^{l} t_{r},
$$

where $v_{d}$ is the volume of a dye molecule.

The efficiency, $\Gamma=\mathcal{W} / \mathcal{E}_{\text {in }}$, from Eqs. (4), (14), and (15), is

$$
\Gamma=\frac{\frac{1}{2} \boldsymbol{\varepsilon}^{d}: \phi_{h d} \mathbf{C}: \boldsymbol{\varepsilon}^{d}}{\left(t_{r} / \tau\right)\left(h v_{d} / v_{d}\right)} f(\gamma) .
$$

The dependence of the light-to-work efficiency $\Gamma$ on material parameters is our second key result. In this form, Eq. (16) enables straightforward interpretation. The quantity $\frac{1}{2} \varepsilon^{d}: \phi_{h d} \mathbf{C}: \boldsymbol{\varepsilon}^{d}$ in Eq. (16) is the work density of a single dye molecule in undergoing strain $\varepsilon^{d}$ in a medium with effective stiffness constant $\phi_{h d} \mathbf{C}$ due to photoisomerization. The quantity $\left(h v_{d} / v_{d}\right)$ in the denominator is the energy density of the photoisomerized dye molecule; $\left(t_{r} / \tau\right)$ is the number of photons absorbed in doing the work. The last term $f(\gamma)$, related to impedance match, is the fraction of mechanical energy that has gone into work on the load. The volume fraction $\phi_{h d}$ depends on illumination as given in Eq. (7), however, it cannot be greater than $\phi_{d}$.

Lack of reliable parameters values makes comparison of experiment and model predictions challenging. Data on highenergy isomer lifetimes $\tau$ and low-energy isomer absorption cross-sections is scarce. Information regarding the effective dye strains $\varepsilon^{d}$ is not readily available. Although the bare dye strain $\varepsilon_{0}^{d}$ can be reasonably estimated from molecular models, the effective dye strain $\varepsilon^{d}=\beta S_{d} \varepsilon_{0}^{d}$ is more difficult to estimate since there is little data available on the orientational order parameter $S_{d}$ and even less on the coupling term $\beta$. Nonetheless, Eq. (16), together with Eq. (7), gives an explicit expression for efficiency in terms of measurable material parameters and illumination.

To enable rough comparison with experiments, we assume that one-half of the dye molecules are in the high-energy conformation, $\phi_{h d}=\phi_{d} / 2$, and $\varepsilon^{d}=2 \varepsilon^{\mathrm{ph}} / \phi_{d}$, and calculating the molecular volume of dyes from mass density and assuming that $f=1 / 2$ and $t_{r}=\tau$, we estimate the efficiency using

$$
\Gamma_{\mathrm{th}} \simeq \frac{\frac{1}{2}\left(\varepsilon^{\mathrm{ph}}\right)^{2} Y}{\phi_{d}\left(h v_{d} / v_{d}\right)}
$$

Using data from publications with adequate photowork information, we provide experimental and theoretical efficiencies for two different liquid crystal elastomers with azo dye and for a two-component organic diarylethene crystal in Table I.

This suggests reasonable agreement between experiment and model predictions, given range of material properties and

\footnotetext{
${ }^{3}$ For simplicity, we do not pursue the anisotropic viscoelastic origins of $t_{r}$ here.
} 
TABLE I. Photowork efficiencies of three photomechanical materials.

\begin{tabular}{lcccccc}
\hline \hline Ref. & Material & $\varepsilon^{\mathrm{ph}}$ & $\phi_{d}$ & $Y(\mathrm{~Pa})$ & $h v_{d} / v_{d}(\mathrm{~Pa})$ & $\Gamma_{\mathrm{ex}}$ \\
\hline$[12]$ & LCE & $6 \times 10^{-2}$ & $1 \times 10^{-3}$ & $1 \times 10^{4}$ & $4 \times 10^{8}$ & $4 \times 10^{-6}$ \\
{$[13]$} & LCN & $8 \times 10^{-3}$ & 1 & $3 \times 10^{8}$ & $6 \times 10^{8}$ & $1 \times 10^{-5}$ \\
{$[7]$} & DAE Crystal & $2 \times 10^{-3}$ & $5 \times 10^{-1}$ & $1 \times 10^{10}$ & $5 \times 10^{8}$ & $7 \times 10^{-6}$ \\
\hline \hline
\end{tabular}

the assumptions made in lieu of missing data. Relevant data to enable more detailed comparison of experiment efficiency and model predictions can be obtained from experiments. ${ }^{4}$

Equation (16) clearly identifies the factors contributing to the efficiency. The dominant contribution to work is $\beta S_{d} \varepsilon_{0}^{d}$ : $\mathbf{C}: \beta S_{d} \varepsilon_{0}^{d}$, where the joint contribution of all terms needs to be maximized. Although this is challenging, the contributing mechanisms have been identified. The volume fraction of the high energy isomer given by Eq. (7) is critical, since due to absorption $\sigma^{l}$ in thick samples can be small, resulting in dramatic reduction of $\phi_{h d}[14,15]$. The energy density of the high energy isomer is given; it represents the maximum energy available for work. Matching system response time to the high energy isomer lifetime is desirable, as is matching load stress to photostress. Current experimental results indicate that bulk of the absorbed light energy goes into heating the sample. Separating thermal and photoinduced contributions to mechanical work are therefore of paramount importance when probing the performance of PM materials.

In the light of the above, we propose, as a basic figure of merit, the ratio of work and energy densities from Eq. (17):

$$
\mathrm{FOM}=\frac{\frac{1}{2}\left(\varepsilon^{\mathrm{ph}}\right)^{2} Y}{\phi_{d}\left(h v_{d} / v_{d}\right)},
$$

which can be readily determined. We mention that results from a different but compatible model are consistent with ours presented here [16]; a detailed comparison will be presented elsewhere. Details of the photomechanical coupling in nematic elastomers have also been studied, including the question of mechanical stability [17].

\footnotetext{
${ }^{4}$ The low-energy isomer absorption cross-section $\mathbf{a}^{l d}$ can be obtained from the absorption coefficient/decay length of the dyed sample. The high-energy isomer lifetime $\tau$ can be obtained from the time dependence of the absorption spectrum of the sample. This allows determination of the characteristic intensity; then from the total dye volume fraction $\phi_{d}$, the volume fraction $\phi_{h d}$ of the high-energy isomer can be obtained as function of incident intensity. Once the volume fraction $\phi_{h d}$ is known, the dye strain can be calculated from the measured photoinduced bulk strain. Knowing the photostress from experiment, the load-to-photostress ratio $\gamma$ can be calculated.
}

\section{CONCLUSION}

In summary, we have identified the appropriate descriptor of the illuminating light, the light stress $\sigma^{l}=\mathbf{E D}$. We have provided a simple model, on the basis of which we have proposed two criteria for assessing the performance of a photomechanical material: the stress gain $\mathbf{R}$, and the work efficiency $\Gamma$. Both can be determined from experimental data. The stress gain is a novel criterion which measures the ratio of light-induced stress in the bulk material relative to the stress from light, and the work efficiency $\Gamma$ measures the ratio of work done on an external load relative to the energy absorbed per dye. We have related both of these quantities to material properties, and showed reasonable agreement between model predictions and experiment. We have identified the material properties of dye stress $\sigma^{d}$ and absorption cross-section tensor $\mathbf{a}^{l d}$ and indicated their role, and indicated how they may be determined from measurements. For the sake of simplicity, we have restricted our attention to the linear regime both in the optical and elastic response, however, the nonlinear optical response in Eq. (7) can be carried forward and large strain elastic response can be used to determine the nonlinear behavior. This is work for the future.

Most importantly, based on insights gained from the model, we identified the individual processes and their contributions to the efficiency of light doing mechanical work. Accordingly, we have indicated strategies to optimize performance related to these processes, and provide direction for improving PM materials performance.

\section{ACKNOWLEDGMENTS}

We acknowledge valuable discussions with D. Broer (Eindhoven University of Technology) and T. Ikeda (Chuo University). We are grateful for collaboration on materials preparation with S.-W. Oh and for useful discussions with R. Hayward (Colorado) and the MURI team. We are grateful for literature references provided by $\mathrm{W}$. Xu. This work was supported by the Office of Naval Research through the MURI on Photomechanical Material Systems (Grant No. ONR N0001418-1-2624).
[1] A. Sánchez-Ferrer, A. Merekalov, and H. Finkelmann, Optomechanical effect in photoactive nematic side-chain liquidcrystalline elastomers, Macromol. Rapid Commun. 32, 671 (2011).

[2] C. L. M. Harvey and E. M. Terentjev, Role of polarization and alignment in photoactuation of nematic elastomers, Eur. Phys. J. E 23, 185 (2007).
[3] C. L. van Oosten, K. D. Harris, C. W. M. Bastiaansen, and D. J. Broer, Glassy photomechanical liquid-crystal network actuators for microscale devices, Eur. Phys. J. E 23, 329 (2007).

[4] C. L. van Oosten, D. Corbett, D. Davies, M. Warner, C. W. Bastiaansen, and D. J. Broer, Bending dynamics and directionality reversal in liquid crystal network photoactuators, Macromolecules 41, 8592 (2008). 
[5] K. M. Lee, D. H. Wang, H. Koerner, R. A. Vaia, L. S. Tan, and T. J. White, Enhancement of photogenerated mechanical force in azobenzene-functionalized polyimides, Angew. Chem., Int. Ed. 51, 4117 (2012).

[6] J. J. Wie, D. H. Wang, K. M. Lee, L. S. Tan, and T. J. White, Molecular engineering of azobenzene-functionalized polyimides to enhance both photomechanical work and motion, Chem. Mater. 26, 5223 (2014).

[7] M. Morimoto and M. Irie, A diarylethene cocrystal that converts light into mechanical work, J. Am. Chem. Soc. 132, 14172 (2010).

[8] F. Terao, M. Morimoto, and M. Irie, Light-driven molecularcrystal actuators: Rapid and reversible bending of rodlike mixed crystals of diarylethene derivatives, Angew. Chem., Int. Ed. 51, 901 (2012).

[9] H. Koshima, R. Matsuo, M. Matsudomi, Y. Uemura, and M. Shiro, Light-driven bending crystals of salicylidenephenylethylamines in enantiomeric and racemate forms, Cryst. Growth Des. 13, 4330 (2013).

[10] M. Dunn, Photomechanics of mono- and polydomain liquid crystal elastomer films, J. Appl. Phys. 102, 013506 (2007).
[11] S-W Oh, T. Guo, A. Kuenstler, R. Hayward, P. Palffy-Muhoray, and $X$. Zheng, Measuring the five elastic constants of a nematic liquid crystal elastomer, Liq. Cryst. 48, 511 (2021).

[12] M. Camacho-Lopez, H. Finkelmann, P. Palffy-Muhoray, and M. Shelley, Fast liquid-crystal elastomer swims into the dark, Nat. Mater. 3, 307 (2004).

[13] M. Yamada, M. Kondo, J. I. Mamiya, Y. L. Yu, M. Kinoshita, C. J. Barrett, and T. Ikeda, Photomobile polymer materials: Towards light-driven plastic motors, Angew. Chem. Int. Ed. 47, 4986 (2008).

[14] D. Corbett and M. Warner, Linear and Nonlinear Photoinduced Deformations of Cantilevers, Phys. Rev. Lett. 99, 174302 (2007)

[15] D. Corbett, C. Xuan, and M. Warner, Deep optical penetration dynamics in photobending, Phys. Rev. E 92, 013206 (2015)

[16] B. Zhou, E. Bernhardt, A. Bhuyan, Z. Ghorbanishiadeh, N. Rasmussen, J. Lanska, and M. G. Kuzyk, Theoretical and experimental studies of photomechanical materials, J. Opt. Soc. Am. B 36, 1492 (2019).

[17] R. Bai and K. Bhattacharya, Photomechanical coupling in photoactive nematic elastomers, J. Mech. Phys. Solids 144, 104115 (2020). 